\title{
Efectos de la contaminación ambiental en la flora y fauna en el cantón La Maná
}

\author{
(c) ()이 (2) \\ BY NC SA \\ Effects of environmental pollution on flora and fauna in the canton of La \\ Maná \\ Pablo Homero Velasteguí López. ${ }^{1}$
}

Recibido: 03-03-2018 / Revisado: 15-03-2018 / Aceptado: 20-03-2018 / Publicado: 05-04-2018

Resumen.

DOI: $\underline{\text { https://doi.org/10.33262/concienciadigital.v1i2.947 }}$

Actualmente, en nuestro país observamos a diario como están las ciudades, contaminadas de residuos sólidos, basura, objetos sin vida, así como también existen factores artificiales como la lluvia ácida y la deforestación ya que esto afecta a la contaminación de la flora y fauna en el cantón.

La contaminación en su definición imposibilita el crecimiento de muchas especies vegetales, porque la presencia de sustancias químicas en el suelo altera los procesos vitales de las plantas. No existe una gran variedad de especies, como ocurre en los sectores no urbanos, donde el hombre ha tenido un menor grado de influencia y son menores los niveles de contaminación.

La consecuencia de las actividades industriales y del transporte, contamina la atmósfera, es responsable de la destrucción de grandes bosques, afecta el suelo que se contamina con sustancias ácidas que dificultan o impiden el crecimiento de nuevas especies vegetales, con lo cual se favorece la erosión del suelo.

La contaminación de la flora y fauna ha provocado la destrucción de la biodiversidad en el Planeta. Actualmente en el Ecuador incluyen aproximadamente el $11 \%$ de las especies existentes en el mundo y el $30 \%$ de las especies enumeradas en América Latina, lo cual en sus datos anteriores era de un 13\%.Tabla 1: Flora y fauna contaminada

\footnotetext{
${ }^{1}$ Ciencia digital, Ambato, Ecuador, pablohomerovelastegui@cienciadigital.org
} 
Actualmente el territorio ecuatoriano recoge 25.000 especies de plantas vasculares. También se ha identificado 2725 especies de orquídeas. El 11\% de las especies existentes en el mundo y el 30\% de las especies enumeradas en América Latina. Los Andes comprenden 1.050 especies, mientras que 850 fueron inventariados en la región amazónica y a lo largo de la costa.

Palabras claves: Contaminación, Biodiversidad, Medio Ambiente, Turismo, Conservación.

\section{Abstract.}

Currently, in our country we see daily how are the cities, contaminated with solid waste, garbage, lifeless objects, as well as artificial factors such as acid rain and deforestation since this affects the contamination of flora and fauna in the canton.

Pollution in its definition makes the growth of many plant species impossible, because the presence of chemical substances in the soil alters the vital processes of plants. There is not a great variety of species, as in the non-urban sectors, where man has had a lesser degree of influence and pollution levels are lower.

The consequence of industrial activities and transport, pollutes the atmosphere, is responsible for the destruction of large forests, affects the soil that is contaminated with acid substances that hinder or prevent the growth of new plant species, which promotes erosion ground.

The contamination of flora and fauna has caused the destruction of biodiversity on the planet. Currently in Ecuador they include approximately $11 \%$ of the existing species in the world and $30 \%$ of the listed species in Latin America, which in their previous data was $13 \%$. Table 1: Flora and fauna contaminated

Currently the Ecuadorian territory collects 25,000 species of vascular plants. It has also identified 2725 species of orchids. $11 \%$ of the existing species in the world and $30 \%$ of the listed species in Latin America. The Andes comprise 1,050 species, while 850 were inventoried in the Amazon region and along the coast.

Keywords: Pollution, Biodiversity, Environment, Tourism, Conservation.

\section{Introducción.}

Según los autores (Parrales, 2004; Benitez, 2009; Luna, 2011) la contaminación ambiental es uno de los problemas más relevantes que nos aqueja diariamente ya que viene afectando nuestro entorno, es más nosotros contribuimos con esta contaminación, ya que perdemos lo bellos que rodea a nuestro Ecuador lo que es la flora y fauna lo cual podría desencadenarse en la destrucción de nuestro planeta tierra. El cantón La Maná, es uno de los siete cantones 
perteneciente a la provincia de Cotopaxi, este bello cantón es muy reconocido por su diversa flora y fauna que existe dentro de ella ya que los turistas lo visitan por sus grandiosos paisajes que lo forman, pero en ello existe una gran preocupación ya que se encuentra en grave peligro de contaminación no sólo de su medioambiente, también la contaminación de sus aguas. Unas de las pérdidas más grandes que ha tenido son los incendios forestales ya que esto afecta a su gran parte de flora y fauna que existe ya que este cantón convive a orillas de La Reserva Ecológica Los Illinizas, en la cual habita gran parte de la flora y fauna que hace llamativo al cantón.

Figura N 1: Flora y fauna contaminada

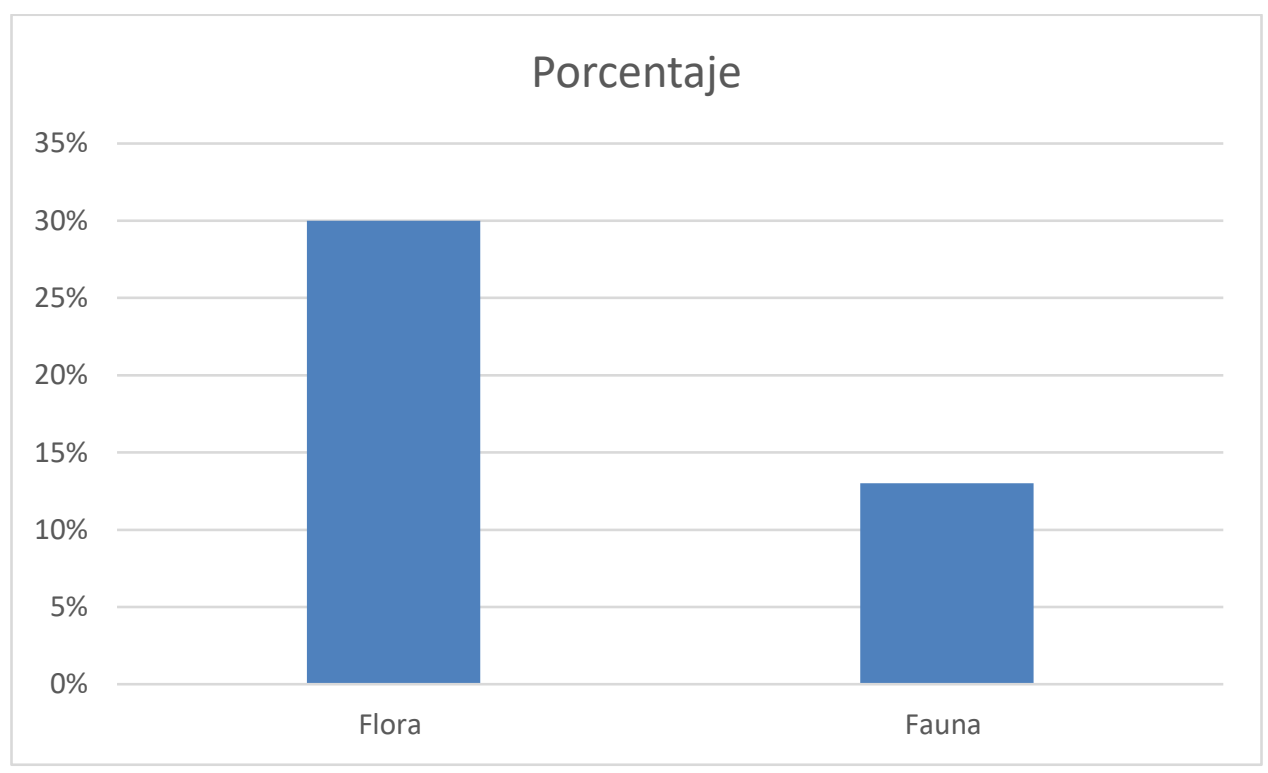

Fuente: https://www.google.com.ec

\section{Criterio Personal}

La contaminación imposibilita el crecimiento de muchas especies vegetales, porque la presencia de sustancias químicas en el suelo altera los procesos vitales de las plantas. Si se observa la vegetación en La Maná, se nota que su ubicación está restringida a los alrededores del sector urbano. No existe una gran variedad de especies, como ocurre en los sectores no urbanos, donde el hombre ha tenido un menor grado de influencia y son menores los niveles de contaminación. Los factores que más afectan a este recurso, provienen de la acción directa del hombre, que produce el llamado efecto antrópico. Los principales problemas ocasionados por la acción humana son: la caza y la pesca indiscriminada, el comercio ilegal de especies animales y la introducción de especies no autóctonas. 


\section{Fauna de la Maná:}

Los autores (Ruiz, 2010; Lopez, 2015; Nuñez, 2015) ya que el cantón es atravesado por la cordillera denominada Nhungañan (ramificación de la cordillera andina) que nace en los Andes, cuenta con una gran variedad de climas, como el subtrópico en el alto de su cordillera hasta el trópico en las partes bajas, donde existen gran variedad de flora y fauna silvestre, su flora se compone de gran variedad de bosques húmedos en el cual habitan gran cantidad de especies. Existen especies de animales en vías de extinción como: el oso de anteojos, Jaguares (últimamente muy poco vistos), tigrillos, pecaris, y dos especies de roedores típicos como la guanta y la guatusa, estos dos últimos víctimas de la caza indiscriminada por parte de cazadores furtivos, entre otras especies de mamíferos, gran variedad de aves (número de especies no contabilizadas, ni estudiadas aun).Como garzas blancas, patos cuervos (grises con franja blanca en el cuello), papagayos, varias especies de loros, perdices, pavas de monte, algunas especies de aves del paraíso en las zonas húmedas, también cuenta con una rica diversidad de reptiles, destacando las serpientes como la falsa coral y la coral, la serpiente equis, entre otras especies venenosas y no venenosas. La contaminación de la flora en el cantón provoca que se pierdan grandes cantidades de orquídeas únicas del lugar, ya que con su gran contaminación imposibilita el crecimiento de muchas especies vegetales, porque la presencia de sustancias químicas tanto en el aire como en el suelo altera los procesos vitales de las plantas. También las actividades industriales y del transporte, contamina la atmósfera y es responsable de la destrucción de grandes bosques. También afecta el suelo, que se contamina con sustancias ácidas que dificultan o impiden el crecimiento de nuevas especies vegetales, con lo cual se favorece la erosión del suelo.

Figura N 2: Fauna de la Maná

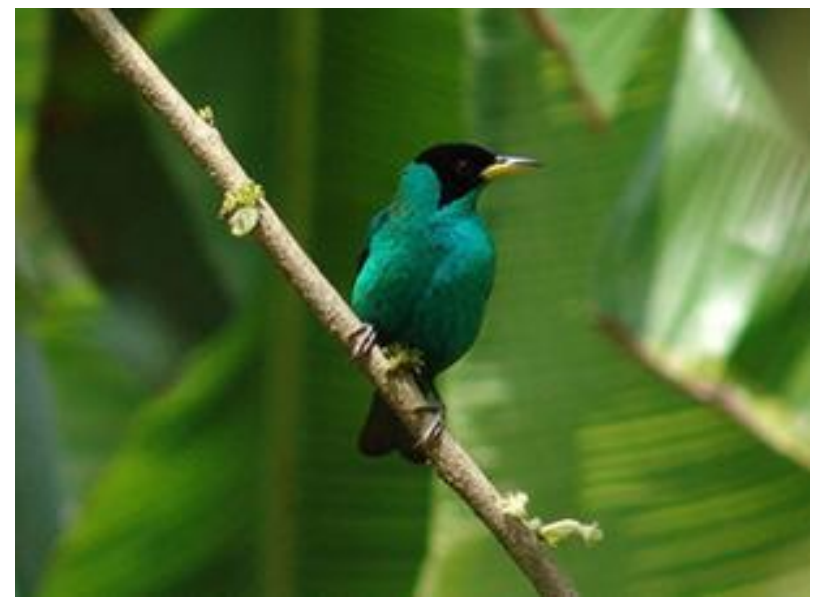

Fuente: https://www.google.com.ec 


\section{Criterio Personal:}

Hay que cuidar nuestro entorno ya que cuando en el suelo se deposita de forma voluntaria o accidental diversos productos como papel, vidrio, plástico, materia orgánica, solventes, plaguicidas, residuos peligrosos o sustancias radioactivas, etc. Probablemente ninguna especie ha tenido el impacto que el ser humano ha logrado sobre la Tierra, y esto se debe principalmente a que ninguna ha alcanzado el nivel de crecimiento poblacional que el ser humano, al crecer de manera geométrica o exponencial provoca que la utilización de recursos naturales también aumenta. Este es un impacto avasallante sobre la biodiversidad, la cual se ve amenazada por varios procesos, la mayoría antropogénicos, que transforman el entorno en el que vivimos.

\section{Practica forestal:}

Lo que los autores (Hidalgo, 2011; Muñoz, 2016; Cela, 2018) las prácticas forestales y agrícolas también afectan así como la deforestación produce el exterminio de diferentes especies vegetales. Un mal manejo de la actividad agrícola también puede alterar el recurso flora, ya que ésta necesita del suelo para vivir y su existencia está fuertemente condicionada a este recurso. Cualquier alteración que sufra el suelo, afectará inevitablemente las especies vegetales. La Fauna también es muy perjudicada ya que la gente siempre ha cazado animales para alimentarse, pero a diferencia de otros depredadores, los seres humanos pueden perseguir a una especie incesantemente hasta que esta se extinga ya que muchos animales soy comercializados o utilizados para usos medicinales. La pérdida del hábitat también pone en peligro a muchos animales. A medida que más y más áreas naturales y silvestres del cantón son desmontadas para construir edificios, rutas, industrias, o para establecer espacios para la agricultura y la ganadería, los animales son aislados y no tienen lugar para moverse, sin sus hábitats ellos se hallan en peligro. Por todo esto y más los seres humanos hemos hecho que tanto la flora y fauna de nuestro cantón se esté extinguiendo y tenemos que tomar conciencia antes de que sea demasiado tarde y provoque una pérdida total de la fauna que existe en ella.

\section{Criterio Personal:}

En lo concerniente a la contaminación de suelos su riesgo es primariamente de salud, de forma directa y al entrar en contacto con fuentes de agua potable. La delimitación de las zonas contaminadas y la resultante limpieza de esta son tareas que consumen mucho tiempo y dinero, requiriendo extensas habilidades de geología, hidrografía, química y modelos a computadora, la presencia de vertidos y acumulación de residuos en lugares no acondicionados, generan una pérdida de calidad del paisaje, a la que se añadiría en los casos más graves el deterioro de la vegetación, el abandono de la actividad agropecuaria y la desaparición de la fauna. Pérdida de valor del suelo económicamente, y sin considerar los costes de la recuperación de un suelo, la presencia de contaminantes en un área supone la 
desvalorización de la misma, derivada de las restricciones de usos que se impongan a este suelo, y por tanto, una pérdida económica para sus propietarios.

\section{Efectos sobre el deterioro de la flora:}

o Mantilla, (2015) la exterminación de las especies vegetales determina una disminución de la cantidad del oxígeno producido por la fotosíntesis, lo que afecta las cadenas tróficas.

- Mantilla, (2015) otro efecto importante, la progresiva erosión que transforma un suelo fértil en un desierto. Lo cual provoca una disminución de flora en el Ecuador, es decir las plantas endémicas se desaparecen.

\section{Efectos sobre el deterioro de la fauna:}

- Mantilla, (2015) afecto en el equilibrio ecológico.

- Mantilla, (2015) la extinción de especies animales o la disminución en su número.

- Mantilla, (2015)destrucción del hábitat de las especies endémicas

- Produce entre ellas una relación de competencia que lleva a la desaparición de unas especies.

○ Mantilla, (2015) rompimiento del delicado equilibrio de la naturaleza.

- Mantilla, (2015) provoca una variación drástica en la cantidad de otras especies pertenecientes a la flora y fauna.

- Mantilla, (2015) la mala explotación del petróleo en la amazonia ha causado la contaminación de ríos por derrames de crudo.

- Mantilla, (2015) por los problemas del mal uso del petróleo ha causado el desplazamiento de etnias autóctonas.

\section{Deterioro de la flora:}

Según (Quiroz, 2010; Mantilla, 2015; Salazar, 2016) el deterioro de la flora, el exterminio de las especies vegetales determina una disminución de la cantidad del oxígeno producido por la fotosíntesis, lo que afecta las cadenas tropicales. Los vegetales son organismos productores de materia orgánica y alimento en las comunidades biológicas por lo mismo, la carencia de la flora incide en el desarrollo de la vida. La consecuencia de la reducción dela flora sobre el ambiente, es la desertificación, es decir, la progresiva erosión que transforma un suelo fértil en un desierto. Este proceso se ve más favorecido aun si la zona en cuestión presenta un clima árido y escasas precipitaciones. Otro efecto importante, consecuencia de la reducción de la flora sobre el ambiente, es la desertificación, es decir, la progresiva erosión que transforma un suelo fértil en un desierto Este proceso se ve más favorecido aun si la zona en cuestión presenta un clima árido y escasas precipitaciones. Además, la no concientización de la población está poniendo en peligro sus propias vidas, porque dónde hay vida, hay gente viviendo, sin embargo, votan en los ríos residuos, en cualquier parte del mundo existe parte 
de la población que ya está acostumbrada a tener una flora muerta, sin vida, ya es hora de cambiar, tomar conciencia, de cómo queremos vivir y en qué condiciones. Entre los principales contaminantes del suelo tenemos la basura, la tala indiscriminada de nuestros árboles, nuestros bosques se desvanecen y con ello todas las especies que las habitan; muchos pobladores talan y queman árboles para comercializar el carbón, sin que las autoridades pongan coto a este problema.

\section{Criterio Personal}

El cambio climático podría afectar el desarrollo de la flora y fauna, fisiología y sus comportamientos durante las fases de crecimiento, reproducción y migración. Por otra parte, es probable también que la modificación en los patrones ya que el cambio climático y sus efectos en la biodiversidad afecten la distribución, tamaño, estructura y abundancia de las poblaciones de algunas especies. Lo anterior, sumado a los efectos del cambio climático sobre el ciclo hidrológico, podría afectar las interacciones entre las especies, los ciclos de nutrientes y el funcionamiento, estructura y distribución misma de los ecosistemas.

\section{Especies de flora y fauna:}

Según (Fajardo, 2008; Davila, 2010; Morales, 2015.)Las especies de flora y fauna, prácticamente desaparecidas en el cantón, es posible actualmente en un centro de recuperación de la biodiversidad de Quito donde se encuentran ejemplares de los legendarios caoba, guayacán, cedros, y variedades de arañas, ranas y aves que se daban por desaparecidas. Como hace unos 10 años atrás podriríamos observar la maravilla de la perfección con aves, animales, mariposas, ranas, árboles gigantes y ríos con aguas muy limpias. Sin embargo, su paulatino proceso de colonización fue destruyendo su entrono lo cual ya no se puede observar fácilmente a simple vista sino que tocaría entrar al interior de las montañas para así poder intentar observarlas. Gran cantidad de turistas y estudiantes de distintas partes del país ingresan a la cuidad para poder observar la biodiversidad que existe en ella por lo que tienen que ingresar a los recintos o comunidades cercanas para poder observarlos y obtener información ya que en la cuidad no se encuentra casi gran cantidad de flora y fauna, los turistas más llegan es por el avistamiento de mariposas ya que es el más nombrado en la cuidad. Por el momento no existe datos precisos de la cantidad de mariposas que existen en el lugar ya que son muchas y en el Ecuador hay un $40 \%$ de especies investigadas y un $60 \%$ de especies por investigar, también su gran cantidad de flora ha desaparecido por la tala de bosques, los incendios forestales, entre otros. Asegura que actualmente conviven entre otros animales, unas 400 variedades de arañas, 50 de ranas, y entre mamíferos, los osos de anteojos, de los que ha "avistado dos ejemplares", asegura, y sostiene que es una especie que se daba por desaparecida en la provincia de Cotopaxi. El exterminio de las especies vegetales determina una disminución de la cantidad del oxígeno producido por la fotosíntesis, lo que afecta las cadenas tróficas. Los vegetales son organismos productores de materia orgánica y 
alimento en las comunidades biológicas; por lo mismo, la carencia de la flora incide en el desarrollo de la vida.

Figura N 3: Plantaciones que perjudican al suelo

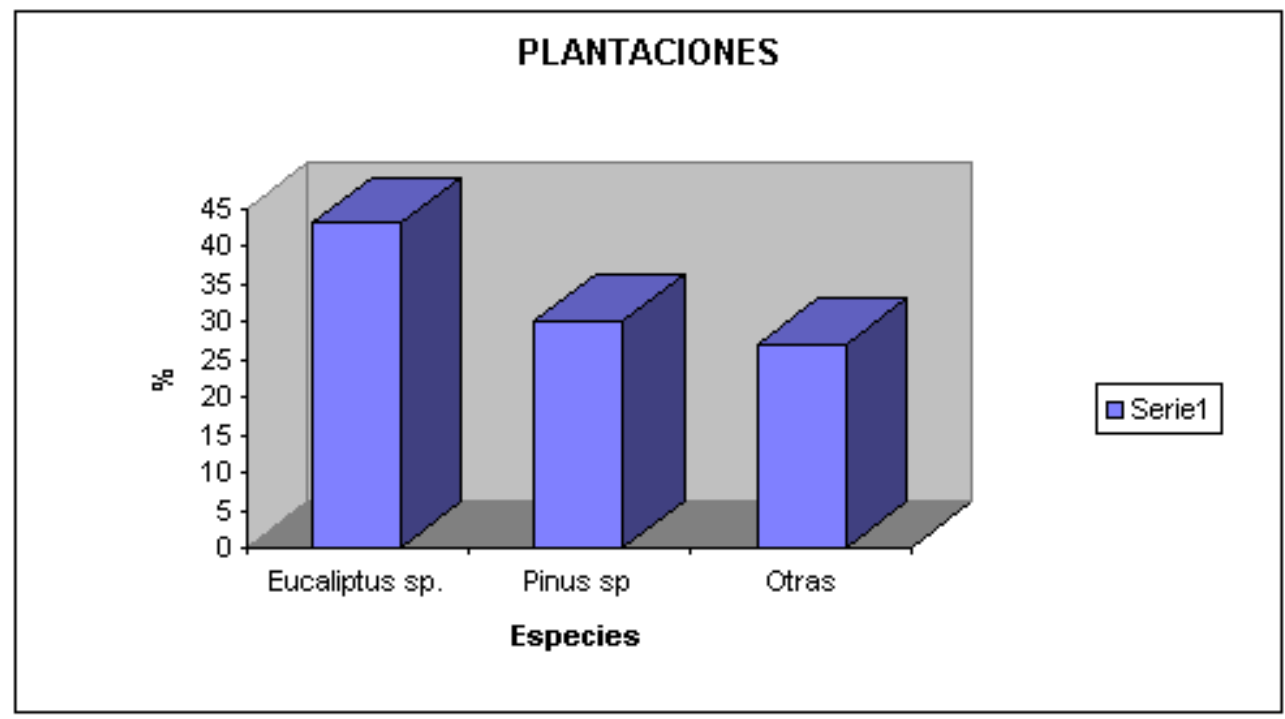

Fuente: https://www.google.com.ec

\section{Criterio Personal:}

La pérdida de especies y el deterioro de los ecosistemas pueden afectar los flujos de bienes y servicios que los ecosistemas proporcionan a la sociedad, la disminución de la cubertura boscosa puede llegar a generar una reducción de la precipitación a escala regional y local, determinante es cualquier factor natural o antrópico que causa, directa o indirectamente, un cambio en un ecosistema o en la biodiversidad. Aquellos que influyen de forma explícita son factores determinantes directos. Entre ellos se encuentran los cambios en el uso del suelo, el cambio climático, la presencia de especies invasoras, la sobreexplotación de los recursos naturales y la contaminación.

\section{Ejemplo de factores que afectan la flora.}

1.- La contaminación: Tuarez, (2016) Imposibilita el crecimiento de especies vegetales, porque la presencia de sustancias químicas en el suelo altera sus procesos vitales. Si se observa la vegetación en Santiago, se nota que su ubicación está restringida a los alrededores del sector urbano. No existe una gran variedad de especies, como ocurre en los sectores no urbanos, donde el hombre ha tenido un menor grado de influencia y es menor la contaminación.

2.- La lluvia acida: Tuarez, (2016) consecuencia de las actividades industriales y del transporte, contamina la atmósfera y es responsable de la destrucción de Bosques. También 
afecta el suelo, que se contamina con sustancias ácidas que dificultan o impiden el crecimiento de nuevas plantas, con lo cual se favorece la erosión del suelo. En Europa se instalaron estaciones medidoras del contenido de ácidos en las lluvias ácidas sobre todo el continente. Así, frente a niveles altos se puede restringir la emisión de los gases industriales.

3.- Las practicas forestales y agrícolas: Tuarez, (2016) la deforestación produce el exterminio de diferentes especies vegetales. Un mal manejo de la actividad agrícola también puede alterar el recurso flora, ya que ésta necesita del suelo para vivir y su existencia está fuertemente condicionada a este recurso. Cualquier alteración que sufra el suelo, afectará inevitablemente las especies vegetales.

\section{Ejemplo de los factores que afectan a la fauna.}

1.- Caza indiscriminada: Tuarez, (2016) la caza indiscriminada ha provocado que varias especies se encuentren en peligro de extinción, tanto a nivel nacional como mundial. Algunas especies afectadas son el culpeo de Tierra del Fuego, la chinchilla chilena y la chinchilla andina.

2.- Pesca indiscriminada: Tuarez, (2016) la intensa actividad pesquera en las costas pone en peligro de extinción las especies marinas. Actualmente, en Chile, la sardina, la anchoveta y el jurel están en serio peligro de desaparecer. Es de gran importancia que se mejoren los mecanismos de explotación de los recursos pesqueros chilenos.

3.- Comercio de especies: Tuarez, (2016) la comercialización y exportación de especies para ser utilizados con fines científicos, para ornamentación o para criadero, han hecho peligrar la fauna de ciertas regiones. Un caso dramático es el del loro amazónico que es sacado de su hábitat para tenerlo encerrado en una jaula.

Figura N 4: Contaminación en la zonas alejadas de la mana

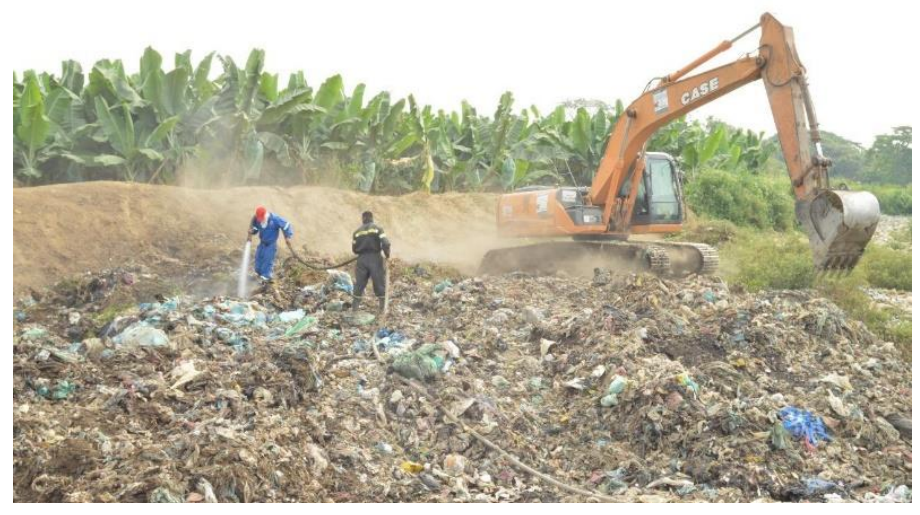

Fuente: https://www.google.com.ec 


\section{Conclusiones}

- La contaminación ambiental en nuestro cantón es un gran problema ya que nadie se quiere responsabilizar por los daños causados en el ambiente, lo cual está afectando a la flora y fauna que existe en ello, por lo cual las plantas dejarían de producir oxígeno y así también se perdería parte de su fauna.

- La flora y fauna en nuestro entorno se ve muy contaminada no solo por la tala de árboles sino también por la extracción de minerales lo cual hace que el uso de químicos en el lugar contamine sus aguas de las cuales están pasan por lugares donde existe gran cantidad de flora y fauna.

- Las variedades de flora y fauna existentes son muy importantes para muchos ya que estos son muy visitados por los turistas, ya que de la cuales existen muchas variedades y formas algunas de ellas aún no han sido investigadas en su totalidad.

- La flora y fauna es una de los atractivos de más demanda en la actualidad, pero en ello existe gran parte que está contaminada lo cual son de varias maneras, las personas no se dan cuenta que están afectando al turismo que tiene el Ecuador

\section{Referencias bibliográficas.}

Benitez, J. L. (2009). Efectos de la contaminación en el Ecuador. Latacunga, Ecuador.

Cela, H. (2018). La biodiversidad de orquides en Cotopaxi, La Maná. Latacunga, Ecuador.

Davila, C. (2010). La perdida de la fauna en la provincia de Cotopaxi. Babahoyo, Los Rios, Ecuador.

Fajardo, H. (2008). La perdida del hábitat de la flora y fauna en la Reserva Ecologica Los Illinizas. Quito, Ecuador.

Hidalgo, R. (2011). Diversas actividades que afectan a la contaminacion ambiental. Quito, Ecuador.

Larcos, M. (2015). El uso de químicos en la explotacion minera. Guayaquil, Ecuador.

Lopez, M. (2015). Flora y fauna que existe en la Reserva Ecologiaca Los Illinizas. Quito, Ecuador.: Editorial Paxi.

Luna, I. (2011). Contaminación en la provincia de Cotopaxi. Loja, Ecuador. 
Malliquinga, E. (2010). Factores de la contaminación ambiental. Latacunga: GAD MUNICIPAL LATACUNGA.

Mantilla, L. (2015). El proceso de exterminio de las especies vegetales en el Ecuador. Baños, Ecuador.

Morales, L. A. (2015.). Causas de la extincion de la flora y fauna en el Ecuador. Lima, Perú.: Universidad Ricardo Palma.

Muñoz, L. (2016). Las practicas forestales como contaminacion del sulo. Lima, Perú.

Nuñez, A. (2015). La variedad de flora y fauna silvestre que esta afectada a causa de la contaminación. Quevedo, Los Rios, Ecuador.

Oto, M. (2014). La perdida de las areas naturales a nivel nacional. Santo Domingo, Ecuador.

Paredes, J. L. (2013). Las consecuencias de las actividades agricolas en la provincia de Cotopaxi. Latacunga, Ecuador.

Parrales, M. E. (2004). Perdida de la flora y fauna en el cantón La Maná. Ambato, Ecuador.

Quiroz, T. (2010). La reducción de la flora en el ambiente. Buenos Aires, Argentina.

Ruiz, M. (2010). Variedad de fauna existente de el Cantón La Maná. Latacunga, Ecuador.

Salazar, J. (2016). La Contaminación Ambiental en el Ecuador. Latacunga, Ecuador.

Tuarez, H. (2016). La contaminación de la flora y fauna en el ambiente. Latacunga, Ecuador.

Zambrano, D. (2015). Las consecuencias de la reduccion de la flora en el cantón La Maná. La Maná, Cotopaxi, Ecauador.: GAD MUNICIPAL DEL CANTÓN LA MANÁ

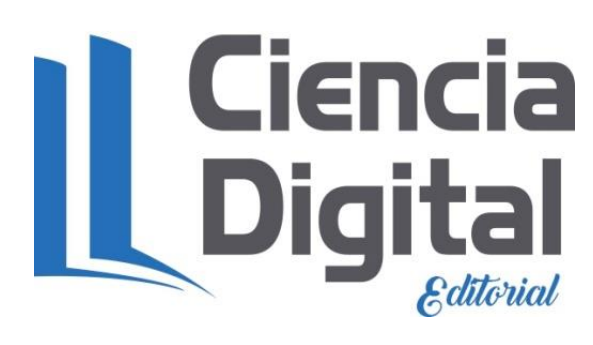


Para citar el artículo indexado.

Velasteguí López, P. (2018). Efectos de la contaminación ambiental en la flora y fauna en el cantón La Maná. ConcienciaDigital, 1(2), 16-27.

https://doi.org/10.33262/concienciadigital.v1i2.947

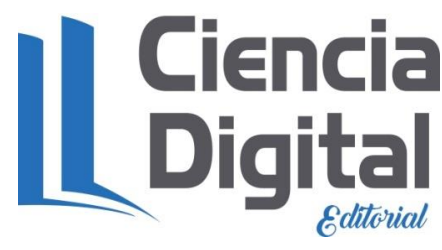

El artículo que se publica es de exclusiva responsabilidad de los autores y no necesariamente reflejan el pensamiento de la Revista Conciencia Digital.

El articulo queda en propiedad de la revista y, por tanto, su publicación parcial y/o total en otro medio tiene que ser autorizado por el director de la Revista Conciencia Digital.
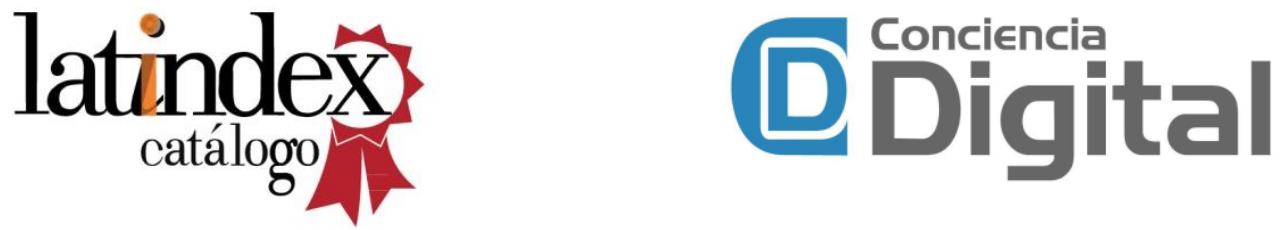